\title{
Preoperative Denosumab may increase the Risk of Local Recurrence of Giant-cell Tumor of Bone Treated with Curettage: A Systematic Review and Meta-analysis
}

\author{
Yongzhao Zhao ${ }^{*}$, Zhenyu Cai*, Xiaodong Tang ${ }^{\bowtie}$, Zhiye Du, Yi Yang, Wei Guo ${ }^{\bowtie}$ \\ Musculoskeletal Tumor Center, Peking University People's Hospital, No. 11 Xizhimen South Street, Xicheng District, Beijing 100044, China. \\ *These authors contributed equally to this study. \\ $\triangle$ Corresponding authors: Xiaodong Tang, MD. Musculoskeletal Tumor Center, Peking University People's Hospital, No. 11 Xizhimen South Street, Xicheng \\ District, Beijing 100044, China. E-mail: tang15877@126.com; OR Wei Guo, MD. Musculoskeletal Tumor Center, Peking University People's Hospital, No. 11 \\ Xizhimen South Street, Xicheng District, Beijing 100044, China. E-mail: bonetumor@163.com.
}

(c) The author(s). This is an open access article distributed under the terms of the Creative Commons Attribution License (https://creativecommons.org/licenses/by/4.0/). See http://ivyspring.com/terms for full terms and conditions.

Received: 2020.07.I I; Accepted: 2020.10.18; Published: 2021.01.0I

\begin{abstract}
Objective: This systematic review and meta-analysis aimed to determine the effect of preoperative denosumab on the local recurrence of giant-cell tumor of bone (GCTB) treated with curettage.

Methods: PubMed, Embase, Cochrane Library, and Web of Science were comprehensively searched. The following data were analyzed using meta-analysis: local recurrence rate of patients receiving denosumab followed by curettage (denosumab group), local recurrence rate of patients receiving curettage only (control group), and a comparison of the local recurrence rates of the two groups.

Results: Nine studies that contained 672 patients with GCTB were included in this review. Patients in the denosumab group (preoperative denosumab followed by curettage) had a higher risk of local recurrence compared with those in the control group (curettage only) (odds ratio $=3.04,95 \%$ confidence interval $=$ I.48-6.22, $P<0.01$ ). The association between preoperative denosumab and local recurrence remained significant in most of the subgroup analyses, except for those with sample sizes $<59(P=0.09)$, sacral GCTB $(P$ $=0.42)$, and usage of postoperative denosumab $(P=0.38)$.

Conclusions: Preoperative denosumab may increase the risk of local recurrence of GCTB treated with curettage and should be used with caution in the management of GCTB.
\end{abstract}

Key words: Denosumab, Giant-cell Tumor of Bone, Local recurrence, Meta-analysis

\section{Introduction}

Giant-cell tumor of bone (GCTB) is a rare primary benign bone tumor that accounts for approximately $5 \%$ of all primary bone tumors [1]. Curettage has become the mainstream therapy for GCTB due to its advantage of preserving the local functional anatomy, such as articular joint surface and nerves. However, the local recurrence rate after curettage remains high despite the usage of local adjuvants (e.g., phenol, peroxide, and liquid nitrogen) [2].

GCTB consists of osteoclast-like giant cells that express the receptor activator of nuclear factor-kappa $\beta$ (RANK) and neoplastic stromal cells that express the RANK ligand (RANKL); RANKL is an indispensable part in the pathogenesis of GCTB [3]. As a full human monoclonal antibody inhibiting RANKL, denosumab has been approved for treating unresectable GCTB or the surgical resection of GCTB that may cause severe morbidity [4, 5]. Previous studies indicated that preoperative denosumab could result in beneficial surgical downstaging in the treatment of GCTB [6]. Data from other studies suggested that preoperative denosumab might increase the risk of local recurrence after the curettage of GCTB [7-15]. Scoccianti et al. [13] evaluated the local recurrence rate of GCTB treated with curettage and cryotherapy, and the authors observed a higher local recurrence rate in preoperative denosumab plus the 
curettage group compared with the curettage-only group $(5 / 12,41.67 \%$ versus $1 / 9,11.11 \%, P<0.05)$. Similarly, Errani et al. [10] observed an increased local recurrence rate in GCTB treated with preoperative denosumab followed by curettage when compared with the curettage-only group (15/25, 60\% versus $36 / 222,16 \%, P<0.05)$. However, Chen et al. [8] indicated a comparable local recurrence rate between preoperative denosumab followed by curettage and curettage-only group in sacral GCTB $(3 / 11,27.27 \%$ versus $3 / 10,30.00 \%)$. A definite conclusion has yet to be obtained about preoperative denosumab on the local recurrence of GCTB treated with curettage because of contradictory results across published studies [7-15]. The current study is a systematic review and meta-analysis that investigates the effect of preoperative denosumab on the local recurrence of GCTB treated with curettage.

\section{Methods}

This study was performed according to Preferred Reporting Items for Systematic Reviews and Meta-Analyses [16], and the protocol of this study was registered in PROSPERO (https://www.crd.york.ac. uk/prospero/) (ID: CRD42020167641).

\section{Eligibility criteria}

The included studies should meet the following inclusion criteria: participants (patients with GCTB), intervention (preoperative denosumab followed by curettage), control (curettage only), outcome (local recurrence), and study design (retrospective or prospective studies). The following studies were excluded: case reports, reviews, animal or cell experiments, inefficient data, non-English language, or duplicated patients.

\section{Information source, literature search, and study selection}

PubMed, Embase, Cochrane Library, and Web of Science were comprehensively searched online on February 6, 2020. The following terms were used in the literature search: ("Giant Cell Tumor" OR "Giant Cell Tumor of Bone" OR "Osteoclastoma") combined with ("AMG162" OR "Denosumab" OR "Xgeva" OR "Prolia"). The details are listed in Supplementary Table S1. The study selection was independently conducted by two investigators according to the eligibility criteria, and any disagreement was resolved through group discussion.

\section{Data collection and summary}

In each study, we extracted the following items: name of the first author, publication year, country, institution, study design, sample size, number of total patients in the denosumab group (preoperative denosumab followed by curettage) or control group (curettage only), local recurrence in denosumab group or control group, tumor site, Campanacci stage [3], previous surgery (primary or recurrent cases), usage of chemical adjuncts during the curettage process, duration of preoperative denosumab, usage of postoperative denosumab, follow-up time, and matched or unmatched factors between the two groups. The duration of preoperative denosumab was transformed from dosage of denosumab if only the dosage was reported in specific studies. Data collection was conducted by two investigators independently, and any disagreement was resolved through group discussion.

\section{Risk of bias in individual studies}

The Newcastle-Ottawa scale (NOS), which has been widely used in meta-analyses [17, 18], was applied to evaluate the risk of bias in the included studies [19]. NOS contains three main categories, namely, selection, scored with four stars; comparability, scored with two stars; and ascertainment of the outcome, scored with three stars. Any study with a score of 1 in the selection or outcome ascertainment, 0 in any of the three domains, or a total score of less than 5 was deemed to have a high risk of bias $[17,18]$.

\section{Statistical analysis}

This study used Review Manager 5.3 software (Cochrane Collaboration, London, UK) and Stata 12.0 (StataCorp, College Station, TX) in the meta-analysis. The association between preoperative denosumab and local recurrence was evaluated using odds ratio (OR) with a corresponding 95\% confidence interval (CI); a P value less than 0.05 indicated a significant association. The local recurrence rate in the denosumab or control group was determined by pooling the data from the included studies. Heterogeneity among the studies was analyzed using the chi-squared test. A fixed-effects model was used in the absence of significant heterogeneity $\left(P>0.10, \mathrm{I}^{2}\right.$ $<50 \%$ ); otherwise, a random-effects model was used $\left(P<0.10, \mathrm{I}^{2} \geq 50 \%\right)$. Subgroup analysis was performed to explore the source of heterogeneity. Sensitivity analysis was carried out to assess the influence of individual studies on the overall results of the association between preoperative denosumab and local recurrence in GCTB treated with curettage. Publication bias across the included studies was evaluated using Egger's test and Begg's test by using Stata 12.0; a $P$ value less than 0.05 indicated a large publication bias. 

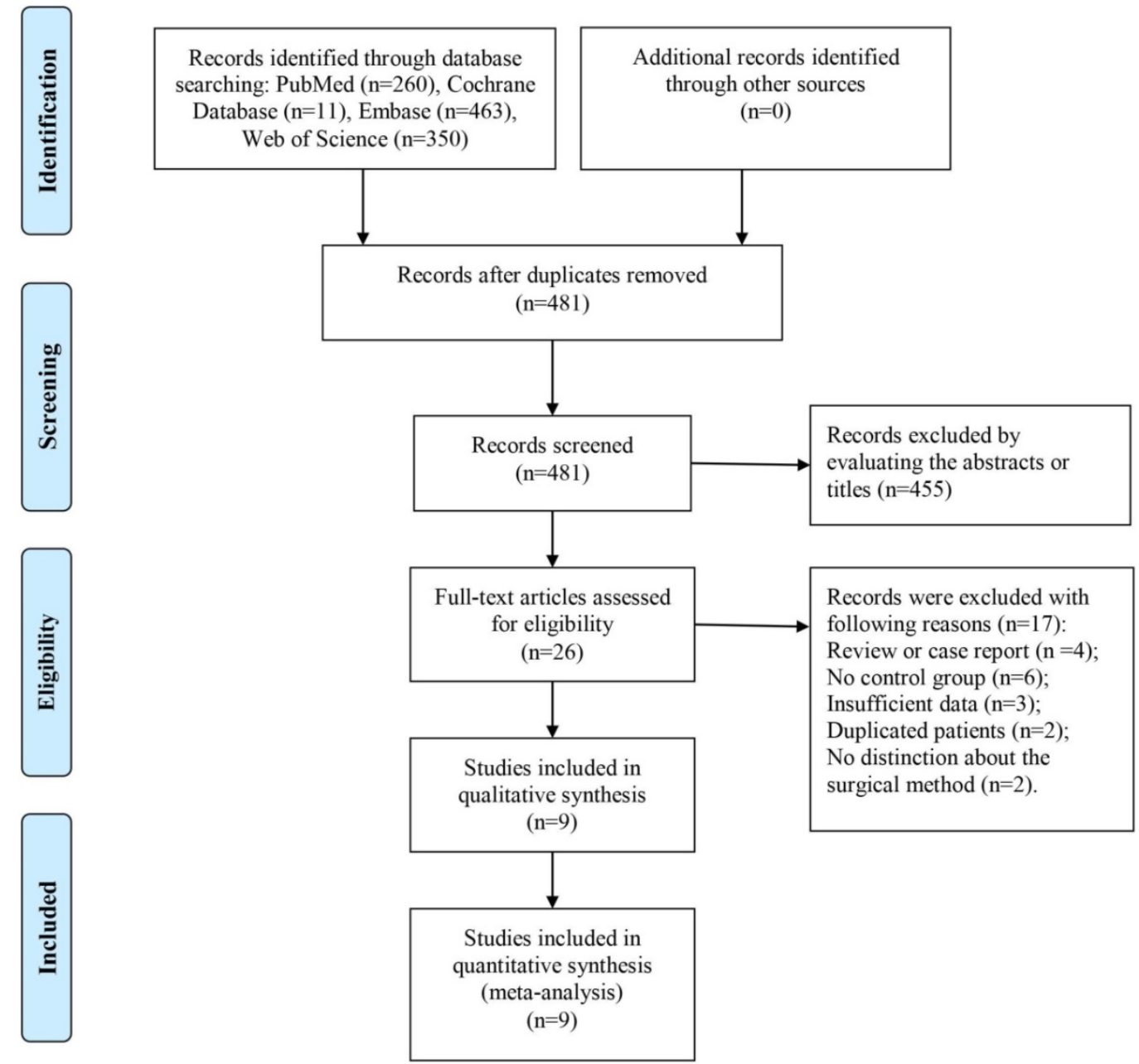

Figure I. Flow chart of the study selection.

\section{Results}

\section{Study selection}

As shown in Figure 1, 1,183 records were obtained from four common databases, namely, PubMed $(n=260)$, Cochrane Library $(n=11)$, Embase $(n=463)$, and Web of Science $(n=350)$. Four hundred and eighty-one records remained after the removal of duplications, and 455 records were directly excluded after the titles or abstracts were scanned. For the remaining 26 records, full texts were carefully evaluated and 17 studies were excluded for the following reasons: review or case report $(n=4)$, no control group $(\mathrm{n}=6)$, insufficient data $(\mathrm{n}=3)$, duplicated patients $(n=2)$, and no distinction about the surgical method (curettage or en bloc resection) (n $=2$ ). Finally, nine studies were included in this systematic review and meta-analysis [7-15].

\section{Characteristics of included studies}

The characteristics of included studies are listed in Table $\mathbf{1}$ and Table 1A. Nine retrospective studies containing 672 patients were included in the analysis
[7-15]. The denosumab group had 172 patients, and the control group had 500 patients. Five studies were performed in Asia [7-9, 14, 15], and the other four studies were performed in Europe [10-13]. Seven studies were conducted in a single medical center $[7-10,12,13,15]$, and two studies were conducted in multiple medical centers $[11,14]$. The sample size varied considerably across the studies from 16 to 247 . With respect to the tumor site, five studies focused on GCTB at all bones $[7,9,11]$, two studies focused on sacral GCTB [8, 15], and two studies focused on appendicular GCTB $[10,12]$. All the studies, except Fedenko et al. [11], reported information on the Campanacci stage and previous surgery of cases. Chemical adjunct was used during the curettage in four studies $[9,10,13,14]$, including phenol, ethanol, and cryotherapy. The median duration of preoperative denosumab ranged from 2 months to 8.9 months, and the patients received both preoperative and postoperative denosumab in three studies $[9,10$, 14]. The median follow-up time ranged from 12 months to 85.6 months among the included studies. Six studies reported the matched factors between the 
two groups, such as age, gender, and Campanacci stage $[7-10,13,15]$. Risk of bias in the studies was evaluated using NOS, and results showed that all the studies had a low risk of bias with scores $>5$ (Table 2).

\section{Local recurrence}

Seventy-two patients suffered from local recurrence in the denosumab group with a local recurrence rate of $43 \%(95 \% \mathrm{CI}=33 \%-57 \%)$, and the fixed-effects model was used in studies without heterogeneity $\left(\mathrm{I}^{2}=0 \%, P=0.57\right)$ (Figure 2A). In the control group, local recurrence occurred in 96 out of 500 patients, and the local recurrence rate was $20 \%$ $(16 \%-25 \%)$ with the fixed-effects model $\left(\mathrm{I}^{2}=0 \%, P=\right.$ 0.57) (Figure 2B).

With regard to the comparison of local recurrence between the denosumab and control groups, the random-effects model was used for evident heterogeneity $\left(\mathrm{I}^{2}=53 \%, P=0.03\right)$. Results showed that the patients in the denosumab group had a significantly higher risk of local recurrence compared with those in the control group $(\mathrm{OR}=3.04$, $95 \% \mathrm{CI}=1.48-6.22, P<0.01)$ (Figure 3). To explore the source of heterogeneity, subgroup analysis was performed in the following factors: ethnicity, sample size, tumor site, Campanacci stage, whether or not a previous surgery was performed, usage of chemical adjunct, duration of preoperative denosumab, and usage of postoperative denosumab. A higher risk of local recurrence was detected in the denosumab group compared with the control group in most analyses $(P<0.05)$, except for sample sizes $<59(P=$ $0.09)$, sacral GCTB $(P=0.42)$, and usage of postoperative denosumab $(P=0.38)$ (Table 3$)$.

Table I. Characteristics of the included studies

\begin{tabular}{|c|c|c|c|c|c|c|c|c|c|c|}
\hline \multirow[t]{2}{*}{ Study } & \multirow[t]{2}{*}{ Country } & \multirow[t]{2}{*}{ Institution } & \multirow{2}{*}{$\begin{array}{l}\text { Study } \\
\text { design }\end{array}$} & \multirow{2}{*}{$\begin{array}{l}\text { Sample } \\
\text { size (n) }\end{array}$} & \multicolumn{2}{|c|}{ Patients (LR/Total) (n) } & \multirow[t]{2}{*}{ Tumor site } & \multicolumn{2}{|c|}{ Campanacci stage (I/II/III) (n) } & \multirow{2}{*}{$\begin{array}{l}\text { Previous } \\
\text { surgery }\end{array}$} \\
\hline & & & & & $\begin{array}{l}\text { Denosumab } \\
\text { group }\end{array}$ & $\begin{array}{l}\text { Control } \\
\text { group }\end{array}$ & & $\begin{array}{l}\text { Denosumab } \\
\text { group }\end{array}$ & Control group & \\
\hline Agarwal et al. 2018 (7) & India & Single center & $\mathrm{R}$ & 59 & $11 / 25$ & $7 / 34$ & $\begin{array}{l}\text { Pelvis, Sacrum, } \\
\text { Extremity }\end{array}$ & $0 / 8 / 17$ & $0 / 9 / 25$ & $\begin{array}{l}\text { Primary, } \\
\text { Recurrence }\end{array}$ \\
\hline Chen et al. 2018 (8) & China & Single center & $\mathrm{R}$ & 21 & $3 / 11$ & $3 / 10$ & Sacrum & $0 / 0 / 11$ & $0 / 0 / 10$ & Primary \\
\hline Chinder et al. 2019 (9) & India & Single center & $\mathrm{R}$ & 123 & $18 / 42$ & $15 / 81$ & Pelvis, Extremities & $0 / 25 / 17$ & $9 / 56 / 16$ & $\begin{array}{l}\text { Primary, } \\
\text { Recurrence }\end{array}$ \\
\hline Errani et al. 2018 (10) & Italy & Single center & $\mathrm{R}$ & 247 & $15 / 25$ & $36 / 222$ & Extremity & $0 / 16 / 9$ & $6 / 173 / 43$ & $\begin{array}{l}\text { Primary, } \\
\text { Recurrence }\end{array}$ \\
\hline Fedenko et al. 2018 (11) & Russian & $\begin{array}{l}\text { Multicenter } \\
\text { study }\end{array}$ & $\mathrm{R}$ & 20 & $6 / 16$ & $2 / 4$ & $\begin{array}{l}\text { Axial skeleton, } \\
\text { Extremity }\end{array}$ & NR & NR & NR \\
\hline Medellin et al. 2018 (12) & $\begin{array}{l}\text { United } \\
\text { Kingdom }\end{array}$ & Single center & $\mathrm{R}$ & 59 & $4 / 4$ & $17 / 55$ & Extremity & $0 / 0 / 4$ & $0 / 32 / 23$ & Primary \\
\hline Scoccianti et al. 2018 (13) & Italy & Single center & $\mathrm{R}$ & 21 & $5 / 12$ & $1 / 9$ & $\begin{array}{l}\text { Pelvis, Sacrum, } \\
\text { Extremity }\end{array}$ & $0 / 4 / 8$ & $0 / 2 / 7$ & Primary \\
\hline Urakawa et al. 2018 (14) & Japan & $\begin{array}{l}\text { Multicenter } \\
\text { study }\end{array}$ & $\mathrm{R}$ & 106 & $6 / 31$ & $15 / 75$ & $\begin{array}{l}\text { Axial skeleton, } \\
\text { Extremity }\end{array}$ & NR & NR & $\begin{array}{l}\text { Primary, } \\
\text { Recurrence }\end{array}$ \\
\hline Yang et al. 2018 (15) & China & Single center & $\mathrm{R}$ & 16 & $4 / 6$ & $0 / 10$ & Sacrum & $0 / 0 / 6$ & $0 / 0 / 10$ & Primary \\
\hline
\end{tabular}

Table I A. Characteristics of included studies.

\begin{tabular}{|c|c|c|c|c|c|c|c|}
\hline \multirow[t]{2}{*}{ Study } & \multirow[t]{2}{*}{ Chemical adjuncts } & \multirow{2}{*}{$\begin{array}{l}\text { Duration of pre- } \\
\text { operative Denosumab } \\
\text { (range) (month) }\end{array}$} & \multirow{2}{*}{$\begin{array}{l}\text { Postoperative } \\
\text { Denosumab (n) }\end{array}$} & \multicolumn{2}{|c|}{ Follow-up time (range) (month) } & \multirow[t]{2}{*}{ Matched factors } & \multirow{2}{*}{$\begin{array}{l}\text { Unmatched } \\
\text { factors }\end{array}$} \\
\hline & & & & $\begin{array}{l}\text { Denosumab } \\
\text { group }\end{array}$ & Control group & & \\
\hline $\begin{array}{l}\text { Agarwal et al. } \\
2018 \text { (7) }\end{array}$ & None & median $3(1-13)$ & None & median $60(27-90)$ & median 27 (12-42) & $\begin{array}{l}\text { tumor site, tumor size, Campanacci } \\
\text { stage, previous surgery }\end{array}$ & NR \\
\hline $\begin{array}{l}\text { Chen et al. } 2018 \\
\text { (8) }\end{array}$ & None & median $2(1-8)$ & 10 patients & mean $18.3(3-36)$ & & Campanacci stage, tumor size & NR \\
\hline $\begin{array}{l}\text { Chinder et al. } \\
2019 \text { (9) }\end{array}$ & $\begin{array}{l}\text { phenol and } \\
\text { ethanol }\end{array}$ & mean $3(1-7)$ & None & mean 32 & mean 37 & $\begin{array}{l}\text { age, gender, symptom, history of } \\
\text { trauma, pathological fracture, tumor } \\
\text { site, tumor size, pulmonary } \\
\text { metastasis, alkaline phosphatase, } \\
\text { calcium, operation time, blood loss, } \\
\text { complication }\end{array}$ & $\begin{array}{l}\text { Campanacci } \\
\text { stage }\end{array}$ \\
\hline $\begin{array}{l}\text { Errani et al. } 2018 \\
(10)\end{array}$ & phenol & median 7 (6-12) & All patients & $\begin{array}{l}\text { median 42.1, } \\
\text { IQR 37.4-50.8 }\end{array}$ & $\begin{array}{l}\text { median 85.6, } \\
\text { IQR 54.3-125.1 }\end{array}$ & $\begin{array}{l}\text { gender, Campanacci stage, previous } \\
\text { surgery }\end{array}$ & $\begin{array}{l}\text { age, tumor } \\
\text { site, phenol }\end{array}$ \\
\hline $\begin{array}{l}\text { Fedenko et al. } \\
2018(11)\end{array}$ & NR & mean 7 & None & median 12.5 & & NR & NR \\
\hline $\begin{array}{l}\text { Medellin et al. } \\
2018 \text { (12) }\end{array}$ & None & mean $8.9(3-19)$ & None & mean $75(12-301)$ & & NR & $\begin{array}{l}\text { Campanacci } \\
\text { stage }\end{array}$ \\
\hline $\begin{array}{l}\text { Scoccianti et al. } \\
2018 \text { (13) }\end{array}$ & cryotherapy & median 7 (4-7) & None & median 39 (14-55) & median 27 (18-92) & $\begin{array}{l}\text { gender, tumor site, cement, bone } \\
\text { graft, plate fixation }\end{array}$ & $\begin{array}{l}\text { Campanacci } \\
\text { stage }\end{array}$ \\
\hline $\begin{array}{l}\text { Urakawa et al. } \\
2018(14)\end{array}$ & $\begin{array}{l}\text { phenol, ethanol or } \\
\text { liquid nitrogen }\end{array}$ & median dosage $6(2-41)$ & 10 patients & NR & & NR & NR \\
\hline $\begin{array}{l}\text { Yang et al. } 2018 \\
\text { (15) }\end{array}$ & None & median 4.5 (3-10) & None & mean $12(7-18)$ & mean 35.3 (13-61) & $\begin{array}{l}\text { age, gender, tumor site, tumor size, } \\
\text { Campanacci stage }\end{array}$ & NR \\
\hline
\end{tabular}

$\mathrm{R}$, retrospective; LR, local recurrence; IQR, interquartile range; NR, not reported. 
Table 2. Risk of bias in the included studies by using the Newcastle-Ottawa scale

\begin{tabular}{|c|c|c|c|c|c|c|c|c|c|}
\hline \multirow[t]{2}{*}{ Study } & \multicolumn{4}{|l|}{ Selection $(* * * *)$} & \multirow{2}{*}{$\begin{array}{l}\text { Comparability }(* *) \\
\text { Comparability on the basis } \\
\text { of design or analysis }\end{array}$} & \multicolumn{3}{|c|}{ Outcome $(* * *)$} & \multirow[t]{2}{*}{ Overall } \\
\hline & $\begin{array}{l}\text { Representativeness } \\
\text { of exposed cohort }\end{array}$ & $\begin{array}{l}\text { Selection of non- } \\
\text { exposed cohort }\end{array}$ & $\begin{array}{l}\text { Ascertainment } \\
\text { of exposure }\end{array}$ & $\begin{array}{l}\text { Outcome not } \\
\text { present at } \\
\text { start }\end{array}$ & & $\begin{array}{l}\text { Assessment } \\
\text { of outcome }\end{array}$ & $\begin{array}{l}\text { Enough } \\
\text { length of } \\
\text { follow-up }\end{array}$ & $\begin{array}{l}\text { Adequacy of } \\
\text { follow up }\end{array}$ & \\
\hline Agarwal et al. 2018 & * & * & * & * & *** & * & * & * & 9 \\
\hline Chen et al. 2018 & * & * & * & * & $*$ & $*$ & & * & 7 \\
\hline Chinder et al. 2019 & * & * & * & * & $*$ & $*$ & * & * & 8 \\
\hline Errani et al. 2018 & * & & * & * & $* *$ & * & * & * & 8 \\
\hline Fedenko et al. 2018 & $*$ & * & * & * & & * & & * & 6 \\
\hline Medellin et al. 2018 & $*$ & * & * & * & & $*$ & * & * & 7 \\
\hline Scoccianti et al. 2018 & $*$ & * & * & * & * & * & * & * & 8 \\
\hline Urakawa et al. 2018 & $*$ & * & * & * & & $*$ & & $*$ & 6 \\
\hline Yang et al. 2018 & $*$ & * & * & * & * & * & & * & 7 \\
\hline
\end{tabular}

A

Study or Subgroup Agarwal et al 2018 Chen et al 2018

Chinder et al 2019

Errani et al 2018

Fedenko and Tararykova 2018

Medellin et al 2018

Scoccianti et al 2018

Urakawa et al 2018

Yang et al 2018

Total $(95 \% \mathrm{Cl})$

Heterogeneity: $\mathrm{Chi}^{2}=6.71, \mathrm{df}=8(\mathrm{P}=0.57) ; \mathrm{I}^{2}=0 \%$
log[Odds Ratio] SE Weight IV.Fixed. $95 \% \mathrm{Cl}$

$\begin{array}{llll}-0.82098 & 0.361814 & 15.5 \% & 0.44\end{array}[0.22,0.89]$

$\begin{array}{lll}-1.29928 & 0.651339 & 4.8 \%\end{array}$

$\begin{array}{lll}-0.8473 & 0.281718 \quad 25.6 \%\end{array}$

$\begin{array}{lll}-0.51083 & 0.326599 & 19.0 \%\end{array}$

$\begin{array}{lll}-0.98083 & 0.478714 \quad 8.9 \%\end{array}$

$\begin{array}{lll}0 & 0.707107 & 4.1 \%\end{array}$

$\begin{array}{lll}-0.87547 & 0.532291 & 7.2 \%\end{array}$

$\begin{array}{lll}-1.64223 & 0.44601 & 10.2 \%\end{array}$

$\begin{array}{ll}-0.40547 & 0.645497\end{array}$

$4.9 \%$

$100.0 \% \quad 0.43[0.33,0.57]$

$0.27[0.08,0.98]$

$0.43[0.25,0.74]$

$0.60[0.32,1.14]$

$0.37[0.15,0.96]$

$1.00[0.25,4.00]$

$0.42[0.15,1.18]$

$0.19[0.08,0.46]$

$0.67[0.19,2.36]$

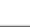

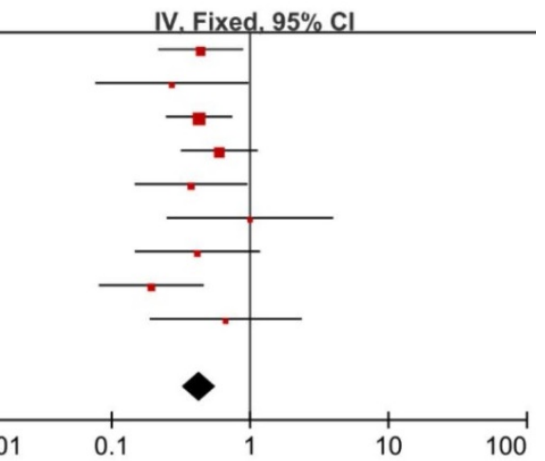

B

Study or Subgroup Agarwal et al 2018 Chen et al 2018

Chinder et al 2019

Errani et al 2018

Fedenko and Tararykova 2018

Medellin et al 2018

Scoccianti et al 2018

Urakawa et al 2018

Yang et al 2018

Total $(95 \% \mathrm{Cl})$

Heterogeneity: $\mathrm{Chi}^{2}=5.71, \mathrm{df}=7(\mathrm{P}=0.57) ; \mathrm{I}^{2}=0 \%$
log[Odds Ratio] SE Weight IV. Fixed, $95 \% \mathrm{Cl}$

$\begin{array}{llll}-1.58045 & 0.415053 & 7.3 \% & 0.21\end{array}[0.09,0.46]$

$\begin{array}{llll}-1.20397 & 0.658281 & 2.9 \% & 0.30\end{array}[0.08,1.09]$

$\begin{array}{llll}-1.6864 & 0.281091 & 15.9 \% & 0.19\end{array}[0.11,0.32]$

$\begin{array}{llll}-1.81916 & 0.179673 & 39.0 \% & 0.16\end{array}[0.11,0.23]$

$\begin{array}{llll}-0.69315 & 0.866025 & 1.7 \% & 0.50[0.09,2.73]\end{array}$

$\begin{array}{llll}-1.17412 & 0.277498 & 16.3 \% & 0.31[0.18,0.53]\end{array}$

$\begin{array}{llll}-2.19723 & 1.054093 & 1.1 \% & 0.11[0.01,0.88]\end{array}$

$\begin{array}{llll}-1.60944 & 0.282843 & 15.7 \% & 0.20\end{array}[0.11,0.35]$

Not estimable

$100.0 \% \quad 0.20[0.16,0.25]$

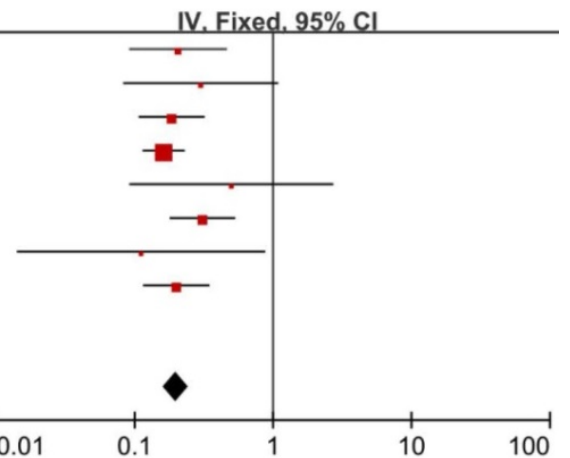

Figure 2. Local recurrence rate (A) denosumab group, (B) control group.

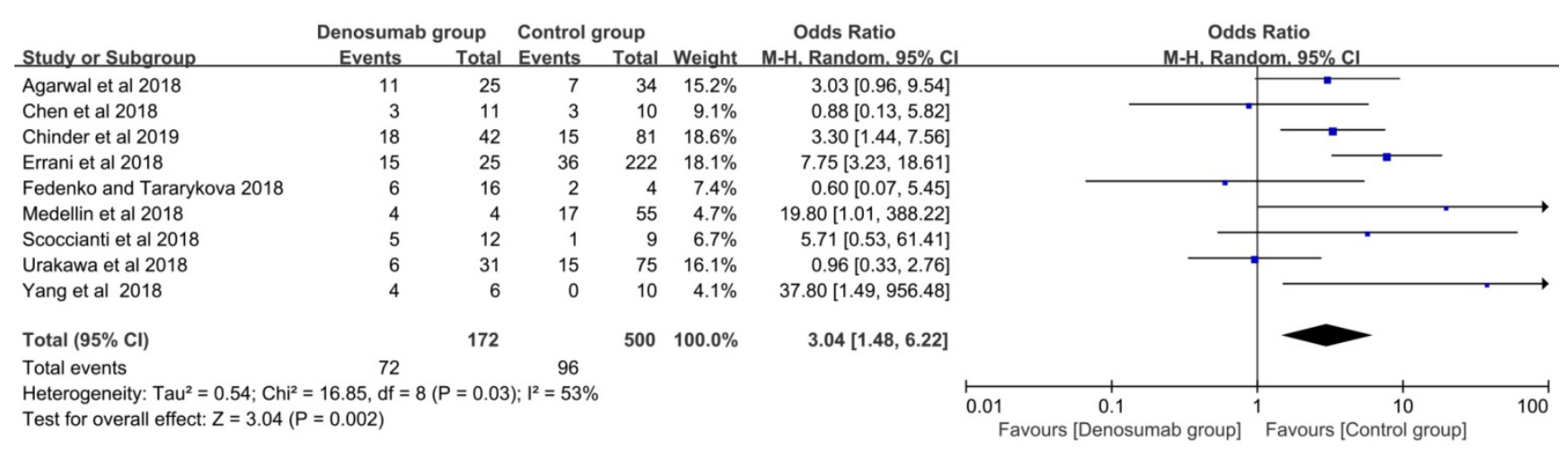

Figure 3. Comparison of local recurrence rate between the denosumab and control groups. 
Table 3. Subgroup analysis in the comparison of local recurrence between the denosumab and control groups

\begin{tabular}{|c|c|c|c|c|c|c|}
\hline Variables & Included Studies & OR $(95 \% \mathrm{CI})$ & $\mathrm{P}$ & $\mathrm{I}^{2}(\%)$ & P for Heterogeneity & Model \\
\hline \multicolumn{7}{|l|}{ Ethnics } \\
\hline Asian & $(7)(8)(9)(14)(15)$ & $2.33(1.40,3.87)$ & $<0.01^{*}$ & 46 & 0.11 & Fixed \\
\hline Caucasian & $(10)(11)(12)$ & $5.67(2.70,11.93)$ & $<0.01^{*}$ & 42 & 0.16 & Fixed \\
\hline \multicolumn{7}{|l|}{ Sample size (n) } \\
\hline$<59$ & $(8)(11)(13)(15)$ & $2.41(0.88,6.56)$ & 0.09 & 49 & 0.12 & Fixed \\
\hline$\geq 59$ & $(7)(8)(10)(12)(14)$ & $3.41(1.51,7.69)$ & $<0.01^{*}$ & 61 & 0.04 & Random \\
\hline \multicolumn{7}{|l|}{ Tumor site } \\
\hline Extremity & $(10)(12)$ & $8.83(3.82,20.40)$ & $<0.01^{*}$ & 0 & 0.54 & Fixed \\
\hline Sacrum & $(8)(15)$ & $4.57(0.11,186.55)$ & 0.42 & 75 & 0.05 & Random \\
\hline Both Axial skeleton and Extremity & $(7)(9)(11)(13)(14)$ & $2.18(1.30,3.65)$ & $<0.01^{*}$ & 28 & 0.24 & Fixed \\
\hline \multicolumn{7}{|l|}{ Campanacci stage } \\
\hline Matched & $(7)(8)(10)(15)$ & $4.28(1.44,12.73)$ & $<0.01^{*}$ & 53 & 0.09 & Random \\
\hline Unmatched & $(9)(12)(13)$ & $4.23(2.02,8.85)$ & $0.02^{*}$ & 0 & 0.49 & Fixed \\
\hline \multicolumn{7}{|l|}{ Previous surgery } \\
\hline Primary & $(8)(12)(13)(15)$ & $4.96(1.69,14.49)$ & $<0.01^{*}$ & 46 & 0.13 & Fixed \\
\hline Both Primary and Recurrence & $(7)(9)(10)(14)$ & $3.04(1.32,7.01)$ & $0.02^{*}$ & 67 & 0.03 & Random \\
\hline \multicolumn{7}{|l|}{ Chemical adjuncts } \\
\hline Yes & $(9)(10)(13)(14)$ & $3.26(1.23,8.59)$ & $0.02^{*}$ & 67 & 0.03 & Random \\
\hline No & $(7)(9)(12)(15)$ & $3.81(1.68,8.66)$ & $<0.01^{*}$ & 46 & 0.13 & Fixed \\
\hline \multicolumn{7}{|l|}{ Preoperative Denosumab (month) } \\
\hline$\leq 3$ & $(7)(8)(9)$ & $2.74(1.46,5.14)$ & $0.02^{*}$ & 0 & 0.45 & Fixed \\
\hline$>3$ & $(10)(11)(12)(13)(15)$ & $6.41(3.13,13.13)$ & $<0.01^{*}$ & 37 & 0.18 & Fixed \\
\hline \multicolumn{7}{|l|}{ Postoperative Denosumab } \\
\hline Yes & $(8)(10)(14)$ & $2.05(0.41,10.18)$ & 0.38 & 81 & $<0.01$ & Random \\
\hline No & $(7)(9)(11)(12)(13)(15)$ & $3.69(2.08,6.55)$ & $<0.01^{*}$ & 19 & 0.29 & Fixed \\
\hline
\end{tabular}

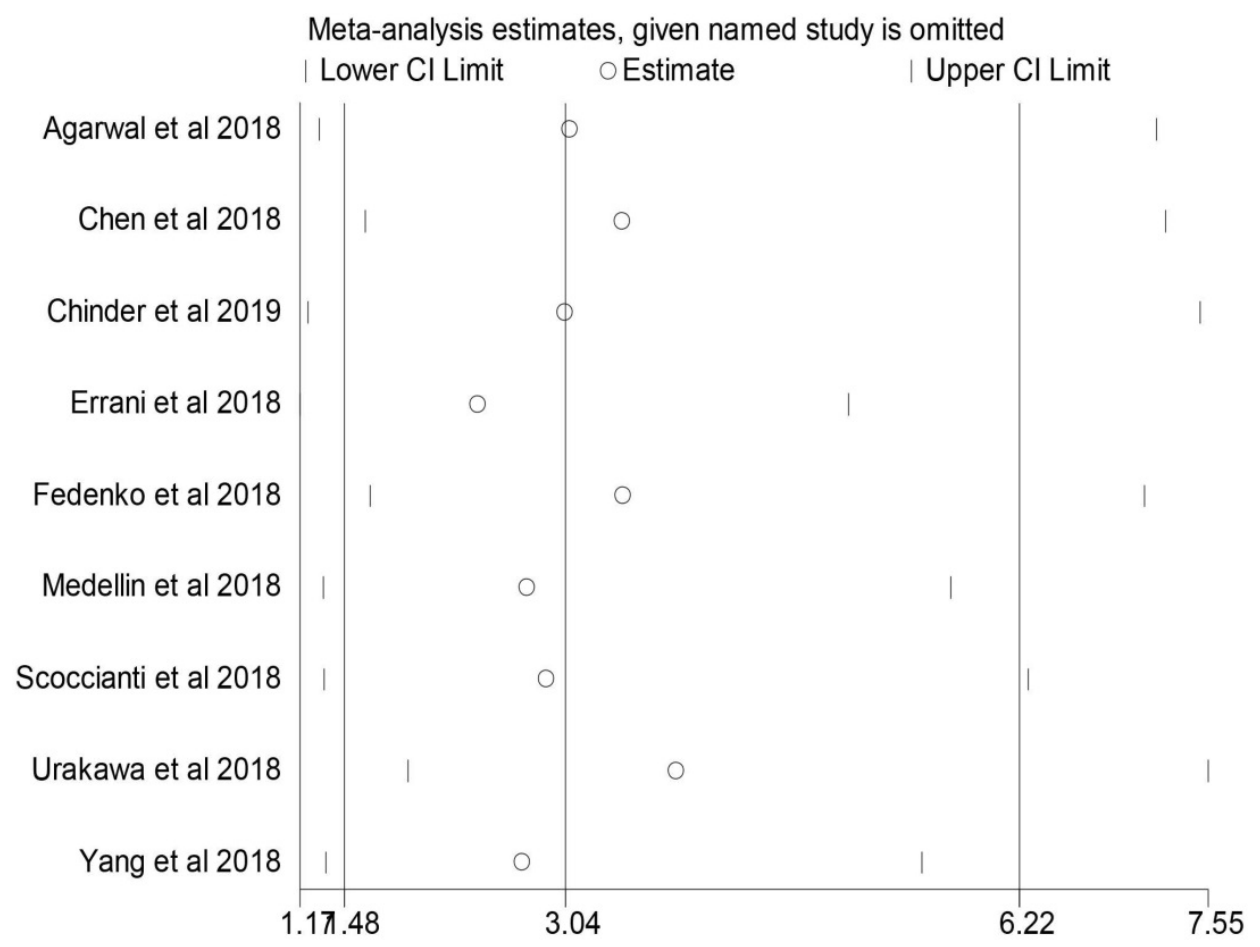

Figure 4. Sensitivity analysis in the comparison of local recurrence rate between the denosumab and control groups.

\section{Sensitivity analysis}

No individual study dominated the overall results of the association between preoperative denosumab and local recurrence in GCTB treated with curettage, and the removal of any single study did not change the overall conclusion (Figure 4).

\section{Publication bias}

No obvious publication bias was observed across the included studies in the meta-analysis of local recurrence between the two groups according to Egger's test $(P=0.19)$ (Figure 5A) and Begg's test $(P=$ 0.08) (Figure 5B). 

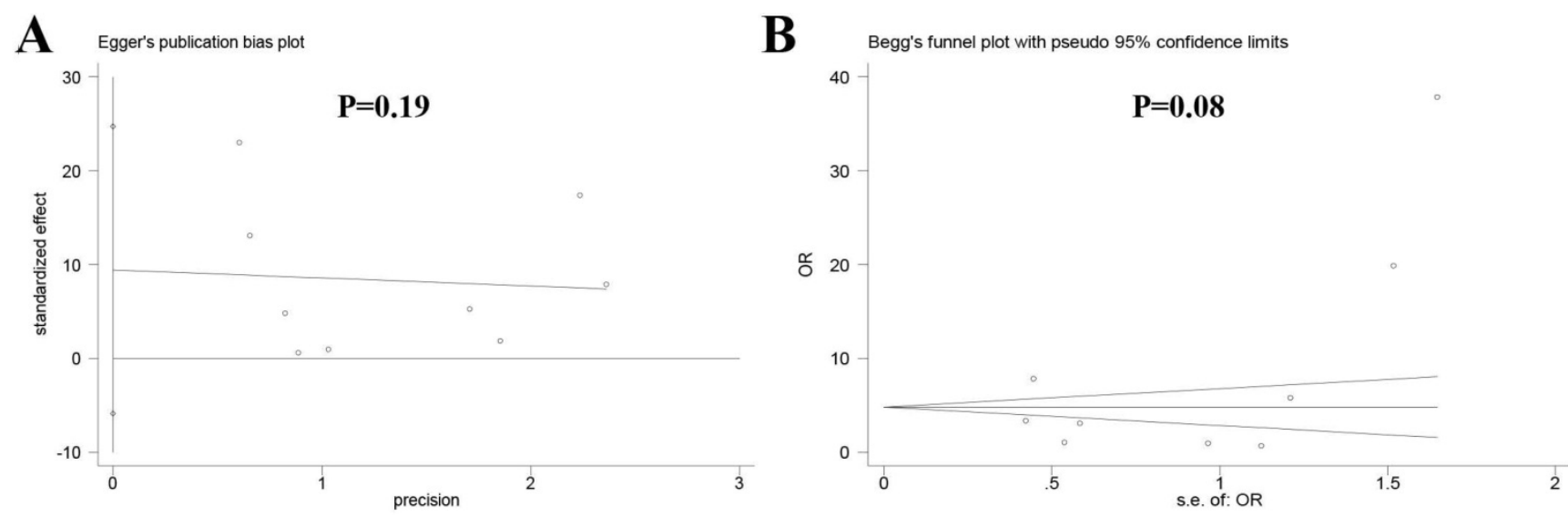

Figure 5. Publication bias among the included studies (A) Egger's test, (B) Begg's test.

\section{Discussion}

The use of denosumab prior to surgical therapy has been gradually accepted in the management of GCTB for surgical downstaging, especially in spinal or sacral GCTB or surgery with a probability of causing severe morbidity [5]. However, increasing evidence has shown that preoperative denosumab might elevate the risk of local recurrence of GCTB after curettage [7-15]. In consideration of the small sample size and contradictory results among published studies [7-15], this systematic review and meta-analysis was performed for the first time to determine the effect of preoperative denosumab on the local recurrence of GCTB treated with curettage.

Several studies have evaluated the local recurrence of GCTB treated with denosumab followed by curettage [20-22]. Niu et al. [20] retrospectively reviewed 13 patients with GCTB who received denosumab followed by curettage with a median follow-up time of 18.8 (range: $10-31$ ) months; $23.08 \%$ (3/13) of patients experienced local recurrence. In Puri et al. [21], $44 \%(11 / 25)$ of patients receiving denosumab followed by curettage suffered from local recurrence during a median follow-up time of 34 (range: 24-48) months. Traub et al. [22] observed a local recurrence rate of $15 \%(3 / 20)$ in patients treated with denosumab followed by curettage with a median follow-up time of 30 (range: 20-45) months. We integrated the data from nine comparative studies in our analysis and found that $43 \%$ of patients receiving preoperative denosumab followed by curettage experienced local recurrence; this value was higher than those in abovementioned studies [20-22]. The high local recurrence rate in our research could be explained by the high proportion of spinal or sacral GCTB $[8,15]$, the non-usage of chemical adjuncts $[7,8$, $12,15]$, short duration of preoperative denosumab [7-9], and potential selection bias of patients $[9,12,13]$.

Denosumab has been widely used in the treatment of GCTB after it was approved by the Food and Drug Administration [23]. Recently, the use of denosumab as a neoadjuvant was attempted in the surgical therapy of GCTB that likely caused severe morbidity (e.g., Campanacci stage 3, severe soft tissue mass, joint resection, and spinal or sacral GCTB), and satisfactory results were obtained. Rutkowski et al. [6] investigated 222 GCTB patients who received preoperative denosumab; $48 \%(106 / 222)$ of patients were saved from surgery, and $38 \%$ (84/222) of patients had a less morbid surgery than originally planned. Although preoperative denosumab might benefit the surgical downstaging of GCTB, accumulating evidence has shown that the usage of preoperative denosumab might increase the risk of local recurrence after curettage [7-15]. In our study, we found that GCTB treated with denosumab followed by curettage was significantly associated with a higher risk of local recurrence compared with curettage only $(43 \%$ versus $20 \%, P<0.01)$. The association between preoperative denosumab and local recurrence was further confirmed by most subgroup analyses $(P<0.05)$, except those with a small sample size $(\mathrm{n}<59)(P=0.09)$, sacral GCTB $(P=$ $0.38)$, and usage of postoperative denosumab $(P=$ $0.09)$. The high risk of local recurrence in patients treated with preoperative denosumab followed by curettage could be explained on the basis of existing evidence. Denosumab could cause irregular ossification within GCTB and form a rim of new bone that possibly contains neoplastic cells [22]. During curettage, the complete removal of the ossification ring of new bone is difficult because of the unclear boundary between the tumor and normal tissues and osteosclerosis of ring. As a result, neoplastic cells remain in the rim of the new bone. Moreover, a translational study demonstrated that denosumab could eliminate only the giant cells in pathological tissues of GCTB, and the surviving stromal cells continue to proliferate in vitro after the withdrawal of 
denosumab [24]. Therefore, the residual neoplastic cells in the ring of new bone might be the main cause of local recurrence after curettage. To overcome these drawbacks, some researchers advised the use of intraoperative C-arm fluoroscopy to distinguish the tumor boundary [7] and the application of ethanol with good penetrability to kill the residual neoplastic cells [25]. However, selection bias may be another reason for the high local recurrence rate in the denosumab group because preoperative denosumab is usually used in complicated cases with a high local recurrence rate, such as patients with Campanacci stage 3, severe soft tissue mass, recurrent cases, and spinal or sacral GCTB [1].

Our findings showed that preoperative denosumab was not obviously associated with the increased risk of local recurrence in patients who received postoperative denosumab $(\mathrm{P}=0.38)$. Postoperative denosumab might delay the local recurrence of GCTB by suppressing the activity of neoplastic cells in the ossification ring caused by preoperative denosumab [24]. Therefore, the usage of postoperative denosumab is probably a feasible choice for delaying the local recurrence of GCTB; however, the optimal duration of postoperative denosumab remains unclear [26]. Our findings also indicate that preoperative denosumab might not increase the risk of local recurrence of sacral GCTB treated with curettage $(\mathrm{P}=0.42)$. Generally, sacral GCTB is difficult to surgically treat because of the surrounding sacral nerves; therefore, preoperative denosumab has been introduced to sacral GCTB in an attempt to decrease the operating difficulty and risk of intraoperative nerve injury [8, 15]. However, our findings on sacral GCTB should be treated with caution because only two studies containing 37 patients were analyzed in this subgroup analysis $[8$, 15]. Moreover, in Chen et al. [8], most patients (10/11) in the denosumab group received postoperative denosumab after curettage, which might delay the local recurrence of GCTB.

We have noticed that Tsukamoto et al. [27] performed a systematic review to explore the role of denosumab in the local recurrence of GCTB treated with curettage. However, several highlights in our study should be noted. First, we pooled the data from the included studies in the form of meta-analysis, which provided convincing evidence on clinical decision-making. Second, researchers focused on the effect of preoperative denosumab instead of postoperative denosumab on the local recurrence of GCTB. Therefore, to eliminate the influence of postoperative denosumab, we tried our best to extract the data of patients who received only preoperative denosumab in the denosumab group and also conducted the subgroup analysis based on the usage of postoperative denosumab. Third, we performed subgroup analysis in our study, which could offer comprehensive evidence on this topic. Fourth, we included two new studies in our analysis, which helped draw a more authoritative conclusion $[8,9]$.

Some limitations should be considered when interpreting our findings. First, all included studies had a retrospective design. As a result, selection bias of patients receiving preoperative denosumab might exist. To reduce the influence of this limitation, we performed subgroup analyses according to tumor site, Campanacci stage, and whether or not the patients received the previous surgery. Second, only nine studies containing 672 patients were included in the analysis, and the relatively small sample size might reduce the persuasiveness of our conclusion. The rarity of this disease might account for this limitation to some extent; multicenter studies should be carried out to deal with this limitation in the future. Third, the duration of preoperative denosumab might be a factor of local recurrence [14]. However, we failed to find the optimal duration of preoperative denosumab because the data of individuals were unavailable. Fourth, many confounding factors, such as surgical technique, chemical adjunct, and invasion of adjacent tissues, were associated with the local recurrence of GCTG [1]. A multivariate analysis should be conducted to determine whether preoperative denosumab is an independent risk factor of local recurrence in future work. Fifth, the follow-up period was relatively short in some included studies $[8,11,15]$, especially in the denosumab group. As a result, the long-term effect of preoperative denosumab on the local recurrence of GCTB after curettage remains unclear. Sixth, although subgroup analyses were performed to detect the source of heterogeneity in the current study, heterogeneity was clear in specific subgroup analyses (e.g., sacral GCTB and usage of postoperative denosumab) and a random-effects model had to be used, which might have lowered the accuracy of the results.

\section{Conclusions}

In conclusion, preoperative denosumab might increase the risk of local recurrence of GCTB treated with curettage. In consideration of our findings, preoperative denosumab should be used with caution in GCTB treated with curettage after the relevant benefits and risks have been balanced adequately. In our center, for patients receiving the curettage therapy, denosumab is tended to be used in patients with high risk of postoperative recurrence or potentially significant surgical morbidity, such as tumors with large soft-tissue extension, close to 
important neurovascular structures or joints of extremities, or large tumors located in the pelvis or spine. Generally, preoperative denosumab $(120 \mathrm{mg})$ is subcutaneously injected on days 1,8 , and 15 , with a loading dosage on day 28 , and every four weeks, if required. Postoperative denosumab (120 mg) is subcutaneously injected monthly in 2 years after the surgery. However, in clinical practice, the duration of preoperative or postoperative denosumab varied a lot because of high expenses and patient's response to the drug [28]. Therefore, multicenter randomized controlled trials should be conducted to further determine the effect of preoperative denosumab on the local recurrence of GCTB treated with curettage and explore the best duration of preoperative denosumab in the future.

\section{Abbreviations}

GCTB: giant-cell tumor of bone; RANK: receptor activator of nuclear factor-kappa $\beta$; RANKL: RANK ligand; NOS: Newcastle-Ottawa scale; OR: odds ratio; CI: confidence interval.

\section{Supplementary Material}

Supplementary table S1.

http://www.jcancer.org/v12p0508s1.pdf

\section{Acknowledgements}

\section{Funding}

This work was supported by the National Natural Science Foundation of China (Grant No. 81972509).

\section{Competing Interests}

The authors have declared that no competing interest exists.

\section{References}

1. Klenke FM, Wenger DE, Inwards CY, Rose PS, Sim FH: Giant cell tumor of bone: risk factors for recurrence. Clin Orthop Relat Res. 2011; 469(2):591-599.

2. van der Heijden L, Dijkstra PD, van de Sande MA, Kroep JR, Nout RA, van Rijswijk CS, Bovee JV, Hogendoorn PC, Gelderblom H: The clinical approach toward giant cell tumor of bone. Oncologist. 2014; 19(5):550-561.

3. Campanacci M, Baldini N, Boriani S, Sudanese A: Giant-cell tumor of bone. J Bone Joint Surg Am. 1987; 69(1):106-114.

4. Chawla S, Blay JY, Rutkowski P, Le Cesne A, Reichardt P, Gelderblom H, Grimer RJ, Choy E, Skubitz K, Seeger L, Schuetze SM, Henshaw R, Dai T, Jandial D, Palmerini E: Denosumab in patients with giant-cell tumour of bone: a multicentre, open-label, phase 2 study. Lancet Oncol. 2019; 20(12):1719-1729.

5. Thomas D, Henshaw R, Skubitz K, Chawla S, Staddon A, Blay JY, Roudier M, Smith J, Ye Z, Sohn W, Dansey R, Jun S: Denosumab in patients with giant-cell tumour of bone: an open-label, phase 2 study. Lancet Oncol. 2010; 11(3):275-280.

6. Rutkowski P, Ferrari S, Grimer RJ, Stalley PD, Dijkstra SPD, Pienkowski A, Vaz G, Wunder JS, Seeger LL, Feng A, Roberts ZJ, Bach BA: Surgical Downstaging in an Open-Label Phase II Trial of Denosumab in Patients with Giant Cell Tumor of Bone. Annals of Surgical Oncology. 2015; 22(9):2860-2868.
7. Agarwal MG, Gundavda MK, Gupta R, Reddy R: Does Denosumab Change the Giant Cell Tumor Treatment Strategy? Lessons Learned From Early Experience. Clin Orthop Relat Res. 2018; 476(9):1773-1782.

8. Chen Z, Yang Y, Guo W, Yang R, Tang X, Yan T, Ji T, Xie L, Xu J, Wang J: Therapeutic benefits of neoadjuvant and post-operative denosumab on sacral giant cell tumor: a retrospective cohort study of 30 cases. J buon. 2018; 23(2):453-459.

9. Chinder PS, Hindiskere S, Doddarangappa S, Pal U: Evaluation of Local Recurrence in Giant-Cell Tumor of Bone Treated by Neoadjuvant Denosumab. Clin Orthop Surg. 2019; 11(3):352-360.

10. Errani C, Tsukamoto S, Leone G, Righi A, Akahane M, Tanaka Y, Donati DM: Denosumab May Increase the Risk of Local Recurrence in Patients with Giant-Cell Tumor of Bone Treated with Curettage. J Bone Joint Surg Am. 2018; 100(6):496-504

11. Fedenko AA, Tararykova A: Neoadjuvant denosumab for the treatment of resectable giant cell tumor of bone: First results of Russian multicenter study. Journal of Clinical Oncology. 2018; 36(15):1.

12. Medellin MR, Fujiwara T, Tillman RM, Jeys LM, Gregory J, Stevenson JD, Parry M, Abudu A: Prognostic factors for local recurrence in extremity-located giant cell tumours of bone with pathological fracture. Bone Joint J. 2018; 100 (-b 12):1626-1632.

13. Scoccianti G, Totti F, Scorianz M, Baldi G, Roselli G, Beltrami G, Franchi A, Capanna R, Campanacci DA: Preoperative Denosumab With Curettage and Cryotherapy in Giant Cell Tumor of Bone: Is There an Increased Risk of Local Recurrence? Clin Orthop Relat Res. 2018; 476(9):1783-1790.

14. Urakawa $\mathrm{H}$, Yonemoto $\mathrm{T}$, Matsumoto $\mathrm{S}$, Takagi $\mathrm{T}$, Asanuma $\mathrm{K}$, Watanuki M, Takemoto A, Naka N, Matsumoto Y, Kawai A, Kunisada T, Kubo T, Emori M, Hiraga H, Hatano H, Tsukushi S, Nishida Y, Akisue T, Morii T, Takahashi M, Nagano A, Yoshikawa H, Sato K, Kawano M, Hiraoka K, Tanaka K, Iwamoto Y, Ozaki T: Clinical outcome of primary giant cell tumor of bone after curettage with or without perioperative denosumab in Japan: from a questionnaire for JCOG 1610 study. World J Surg Oncol 2018, 16(1):160.

15. Yang Y, Li Y, Liu W, Xu H, Niu X: A nonrandomized controlled study of sacral giant cell tumors with preoperative treatment of denosumab. Medicine (Baltimore) 2018, 97(46):e13139.

16. Moher D, Liberati A, Tetzlaff J, Altman DG: Preferred reporting items for systematic reviews and meta-analyses: the PRISMA statement. Bmj. 2009; 339:b2535.

17. Wallis CJ, Mahar AL, Choo R, Herschorn S, Kodama RT, Shah PS, Danjoux C, Narod SA, Nam RK: Second malignancies after radiotherapy for prostate cancer: systematic review and meta-analysis. Bmj. 2016; 352:i851.

18. Viale L, Allotey J, Cheong-See F, Arroyo-Manzano D, McCorry D, Bagary M, Mignini L, Khan KS, Zamora J, Thangaratinam S: Epilepsy in pregnancy and reproductive outcomes: a systematic review and meta-analysis. The Lancet. 2015; 386(10006):1845-1852.

19. Wells GA, Shea B, O'Connell D, Peterson j, Welch V, Losos M, Tugwell P: The Newcastle-Ottawa Scale (NOS) for Assessing the Quality of Non-Randomized Studies in Meta-Analysis. 2000; www.ohri.ca/programs/clinical_epidemiology/oxford.asp.

20. Niu X, Yang Y, Wong KC, Huang Z, Ding Y, Zhang W: Giant cell tumour of the bone treated with denosumab: How has the blood supply and oncological prognosis of the tumour changed? J Orthop Translat. 2019; 18:100-108.

21. Puri A, Gulia A, Hegde P, Verma V, Rekhi B: Neoadjuvant denosumab: its role and results in operable cases of giant cell tumour of bone. Bone Joint J. 2019; 101(-b 2):170-177.

22. Traub F, Singh J, Dickson BC, Leung S, Mohankumar R, Blackstein ME, Razak AR, Griffin AM, Ferguson PC, Wunder JS: Efficacy of denosumab in joint preservation for patients with giant cell tumour of the bone. Eur J Cancer. 2016; 59:1-12.

23. Kaufman M: Pharmaceutical Approval Update. P \& T : a peer-reviewed journal for formulary management. 2017; 42:673-683.

24. Mak IW, Evaniew N, Popovic S, Tozer R, Ghert M: A Translational Study of the Neoplastic Cells of Giant Cell Tumor of Bone Following Neoadjuvant Denosumab. J Bone Joint Surg Am. 2014; 96(15):e127.

25. Gaston CL, Grimer RJ, Parry M, Stacchiotti S, Dei Tos AP, Gelderblom H, Ferrari S, Baldi GG, Jones RL, Chawla S, Casali P, LeCesne A, Blay J-Y, Dijkstra SPD, Thomas DM, Rutkowski P: Current status and unanswered questions on the use of Denosumab in giant cell tumor of bone. Clinical Sarcoma Research. 2016; 6(1):15.

26. Palmerini E, Chawla NS, Ferrari S, Sudan M, Picci P, Marchesi E, Leopardi MP, Syed I, Sankhala KK, Parthasarathy P, Mendanha WE, Pierini M, Paioli A, Chawla SP: Denosumab in advanced/unresectable giant-cell tumour of bone (GCTB): For how long? European Journal of Cancer. 2017; 76:118-124. 
27. Tsukamoto S, Tanaka Y, Mavrogenis AF, Kido A, Kawaguchi M, Errani C: Is Treatment with Denosumab Associated with Local Recurrence in Patients with Giant Cell Tumor of Bone Treated with Curettage? A Systematic Review. Clin Orthop Relat Res. 2019.

28. Lim CY, Liu X, He F, Liang $H$, Yang $Y$, Ji T, Yang $R$, Guo W: Retrospective cohort study of 68 sacral giant cell tumours treated with nerve-sparing surgery and evaluation on therapeutic benefits of denosumab therapy. Bone Joint J. 2020; 102 (-b 2):177-185. 